\title{
Electronic Warning System Based on Surface Acoustic Wave Vibration Sensors
}

\author{
J. Filipiak ${ }^{a, *}$, L. SOlARZ ${ }^{b}$ AND G. SteCZKO ${ }^{a}$ \\ ${ }^{a}$ Institute of Electronic and Control Systems, Technical University of Częstochowa \\ Armii Krajowej 17, 02-240 Częstochowa, Poland \\ ${ }^{b}$ Department of Civil Engineering, Military University of Technology \\ S. Kaliskiego 2, 00-908 Warsaw, Poland
}

\begin{abstract}
In the paper feasibility analysis of the surface acoustic wave based electronic warning system was presented. As a transducer in the system the surface acoustic wave delay line was proposed. It is fabricated in standard single mask process as a pair of interdigital transducers placed on the top of piezoelectric crystal substrate. Transducer is a plate with rectangular cross-section and it converts all environmental vibration to electric signal. From electronic point of view it was considered as a two-port network. Necessary condition for proper sensor operation is that the input signal frequency from external generator should be close to central frequency of delay line. Principle of operation of the sensor is that the measured phase shift of high frequency signal has frequency equal resonant frequency of vibrating plate and its amplitude is proportional to vibration amplitude of transducer. The environmental vibrations are defined by amplitude of the output signal and the threat location is pointed by resonant frequency of excited transducer. The described above principles of sensor operation allow us designing electronic warning system consisting of many sensors with different resonant frequencies of transducers. Output signals from precisely located sensors contain all necessary information about the whole warning system. This information is easy to transmit and detect by simple electronic circuits based on phase detector and proper data acquisition system, for which necessary conditions are presented.
\end{abstract}

PACS: 77.65.Dq, 68.35.Iv

\section{Introduction}

Airports, petrol depot or dangerous chemicals stores are high risk objects. Thus technical security system for such objects consists in detection and localization of all possible threats is very important and can be a perimeter system. This type of system is a base to design an electronic warning system architecture by means of a linear surface acoustic wave vibration sensor (SAW-VS). Specially packaged sensors placed on the enclosures or underground and connected together by simple electronic elements form electronic warning system. It is able to detect presence of intruder in close of the SAW-VS surrounding. The most important part of the system is the SAW-VS [1] and its schematic diagram is shown in Fig. 1.

The main part of the SAW-VS is an anisotropic piezoelectric plate, one site clamped to the package and second free vibrating and mass loaded. On the top of the plate two interdigital transducers (IDTs) form the SAW delay line. This pair of IDTs are an electronic filter with specially selected central frequency (e.g. $\Omega_{0}=78 \mathrm{MHz}$ ) and described as a four-terminal network. Electronic signal with that frequency excites surface acoustic wave in one

* corresponding author; e-mail: gem@gemsc.com.pl

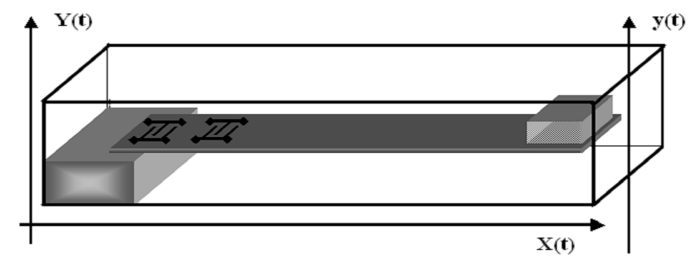

Fig. 1. Schematic diagram of the SAW-VS.

IDT and the second receives it. Sensor package movement causes vibrations of the piezoelectric plate, and simultaneously changes time delay of propagated SAW. This effect causes phase shift of input high frequency signal. Phase shift is time dependent signal and its amplitude is proportional to time delay changes of the SAW and frequency is equal to resonant frequency of piezoelectric plate. As was shown in Refs. [1-3] the SAW-VS has only one normal frequency and it is dependent on its geometry. This allows us identifying any SAW-VS in a system and immediate thread localization. Summarizing phase shift of high frequency signal depends on vibration amplitude of piezoelectric plate and its resonant frequency and these two parameters describe a state of ground or enclosure. 
Measurement of mentioned above parameters, coded in high frequency signal, is difficult. One of practical configuration of measurement system is shown in Fig. 2.

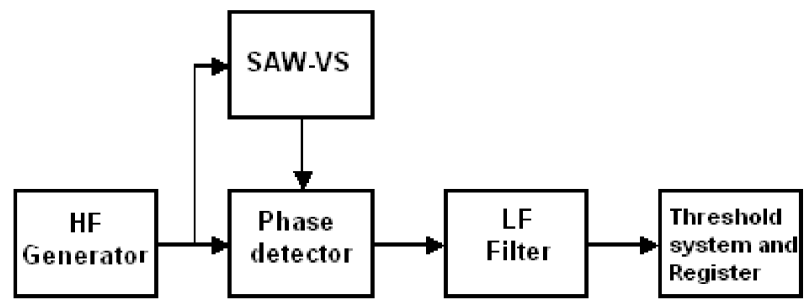

Fig. 2. Schematic diagram of detection method in the SAW-VS.

It consisted of high frequency generator (HFG), the SAW-VS, phase detector (PD), low frequency filter (LF) and data acquisition (DAQ) system. In this method vibration amplitude and frequency are changed to time dependent signal. Such kind of signal is easy to acquire and secure real time information about state of the SAW-VS and it continues vibrations monitoring of defined protection zone. Basic condition which is to be fulfilled is that DAQ detected signal must be proportional to normal vibration of the sensor piezoelectric plate. It requires detailed analysis cooperation of several SAW-VSs and electronic measurement system from Fig. 2.

Single SAW-VS is a four-terminal network which allows us to cascade connection of several SAW-VSs. The principles of measurement method do not change if electronic parameters of SAW-VSs are precisely selected. They must have the same central frequency of SAW delay line and different resonant frequencies. If losses of the SAW filter are compensated by amplifier then amplitude of high frequency signal will pass through all sensors without any changes. But phase shift of this signal is a sum of phase shifts from the whole set of sensors. Thus the phase shift carries information about vibrations of all SAW-VSs in a system. Measured amplitude changes of SAW delay line gives possibility to find vibration parameters which comes from surroundings of any sensor. Frequency response of phase shift allows us to localize any sensor because of its particular resonant frequency. Taking into account all above it is possible to design electronic warning system by cascade connection of several SAW-VSs with measurement method from Fig. 2.

\section{Operation analysis of electronic warning system}

Electronic warning system considered in the paper is shown in Fig. 3. It consists of five SAW-VSs placed in two paths and numbered $1-5$, high frequency generator (HFG) and phase detector (PD).

Principle of operation for such system is as follows. Signal from high frequency generator

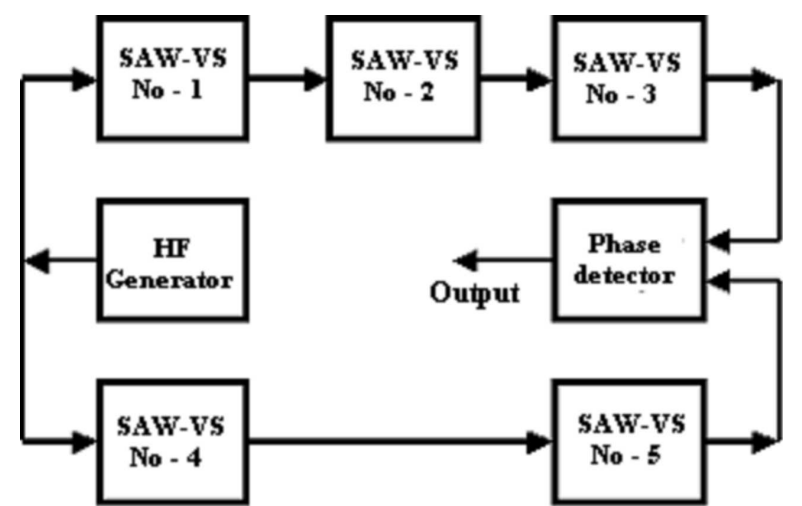

Fig. 3. Schematic diagram electronic warning system with five SAW-VSs.

$$
u(t)=U \sin \left(\Omega_{0} t\right),
$$

where $U$ - amplitude, $\Omega_{0}$ - frequency HFG, is divided into two paths. First path consists of three cascade connected SAW-VSs numbered 1-3 and second path with sensors 4 and 5 . Input signals of the phase detector are

$$
\begin{aligned}
& u_{1}(t)=U_{1} \sin \left(\Omega_{0} t+\Psi_{1}(t)\right), \\
& u_{2}(t)=U_{2} \sin \left(\Omega_{0} t+\Psi_{2}(t)\right),
\end{aligned}
$$

where $U_{1}, U_{2}, \Psi_{1}(t), \Psi_{2}(t)$ - amplitude and phase shifts for two paths, respectively.

Total phase shifts are a sum of phase shifts from sensors, electronic elements and wires. Output signal from phase detectors takes the form

$$
\begin{aligned}
& U_{\mathrm{d}}(t)=\frac{1}{2} k_{m} U_{1} U_{2} \cos \left(\Psi_{1}-\Psi_{2}\right) \\
& +\frac{1}{2} k_{m} U_{1} U_{2} \cos \left(2 \Omega_{0} t+\Psi_{1}+\Psi_{2}\right),
\end{aligned}
$$

where $k_{m}$ - constant values of phase detector.

From measurement point of view only first term of relation (3) is interesting. It has slow time variation compared to generator signal and its frequency is proportional to phase difference input signals. Second term is removed by low frequency filter.

Signal after filtering is as follows:

$$
\begin{aligned}
& u_{\mathrm{d}}(t)=k_{\mathrm{d}} \cos \Psi_{\mathrm{b}}, \\
& k_{\mathrm{d}}=\frac{1}{2} k_{m} U_{1} U_{2},
\end{aligned}
$$

where $\Psi_{\mathrm{b}}=\Psi_{1}-\Psi_{2}$. Relation (4) is called phase detector characteristic. It depends on phase difference and amplitudes of input signals. It is the function with period $2 \pi$ and coefficient $k_{\mathrm{d}}$ defines slope in point $\Psi_{\mathrm{b}}=\pi / 2$ and it is called working point of the phase detector. Assuming negligible amplitudes and small fluctuation of phase of the input signals around working point, output signal takes the form

$$
u_{\mathrm{d}}(t)=k_{\mathrm{d}}\left(\Psi_{1}-\Psi_{2}\right) .
$$

By assuming that phase shifts in both paths originate 
from times delay of SAW filters and other sources of phase shift can be compensated, phase shifts in both paths can be expressed as

$$
\begin{aligned}
& \Psi_{1}=\sum_{n=1}^{3} \varphi_{n}, \\
& \Psi_{2}=\sum_{n=4}^{5} \varphi_{n},
\end{aligned}
$$

where

$$
\varphi_{n}=2 \pi \Omega_{0} \Delta \tau_{n},
$$

$\varphi_{n}$ is phase shift of $n$-th sensor, $\Delta \tau_{n}$ is time delay changes of $n$-th SAW filter.

Further analysis requires explicit definition time delay of the SAW as a function amplitude of vibration and resonant frequency of piezoelectric plate for any sensor in the system.

\section{Analysis of the SAW-VS}

Time delay changes $\Delta \tau$ of the SAW in particular sensor is caused by

- SAW velocity changes affected by stress of vibrated plate,

- propagation length changes of the SAW affected by deformation of the plate.

Full description of time delay changes $\Delta \tau$ as a function of the sensor movement defined by mentioned above two parameters is very complicated. Anisotropic model of piezoelectric plate has to be applied and time delay has only numerical form as was shown in Refs. [2, 3]. But for design electronic warning system it was assumed that time delay changes $\Delta \tau$ is proportional to deflection of end of the plate, i.e.

$$
\Delta \tau_{n} \propto y(t) .
$$

Taking into account relation (8), it implicates directly that phase shift of any sensor is proportional to plate end's deflection, too

$$
\varphi_{n} \propto y(t) \text {. }
$$

This assumption allows us applying one degree of freedom model to model vibration piezoelectric plate [2-6]. Plate vibrations are forced by movement of sensor package along $Y$ axis and sensor does not move in $X$ axis. Equation of motion takes the form

$$
\frac{\mathrm{d}^{2} y(t)}{\mathrm{d} t^{2}}+\omega_{0}^{2} \tau \frac{\mathrm{d} y(t)}{\mathrm{d} t}+\omega_{0}^{2} y(t)=\frac{\mathrm{d}^{2} Y(t)}{\mathrm{d} t^{2}},
$$

where $y(t)$ - function defining end of plate movement, $Y(t)$ - function defining sensor package movement, $\tau$ equivalent damping coefficient of plate material [3], $\omega_{0}$ - angular resonant frequency of non-damped vibrations of the plate.

The angular resonant frequency of the plate is

$$
\omega_{0}=3.5172\left(\frac{h}{l^{2}}\right) \sqrt{\frac{E_{\mathrm{e}}}{12 \rho}} \sqrt{\frac{1}{1+r 3.9689}},
$$

where $l$ - length of the plate, $h$ - height of the plate, $\rho$ - density of the plate, $r$ - seismic mass to mass of the plate ratio, $E_{\mathrm{e}}$ - equivalent Young module [3].

Analytical solution for Eq. (11) is

$$
\begin{aligned}
& y(t)=A \exp \left(-\frac{\omega_{0}^{2} \tau t}{2}\right) \sin \left(\omega_{r}(t+\alpha)\right) \\
& -\frac{1}{\omega_{r}} \int_{0}^{t} \ddot{Y}(\xi) \exp \left(-\frac{\omega_{0}^{2} \tau}{2}(t-\xi)\right) \sin \left(\omega_{r}(t-\xi)\right) \mathrm{d} \xi,
\end{aligned}
$$

where

$$
\omega_{r}=\omega_{0} \sqrt{1-\frac{\omega_{0}^{2} \tau^{2}}{4}} .
$$

$\omega_{r}$ - angular frequency of vibrations. Constant values $A$ and $\alpha$ are calculated from initial conditions.

From mathematical point of view phase shift of high frequency signal, which passes through sensor, is proportional to

- convolution impulse response of plate and acceleration component in the $Y$ direction,

- free damped vibration function

$$
y_{\delta}(t)=A \mathrm{e}^{-\frac{\omega_{0}^{2} \tau}{2} t} \sin \left(\omega_{r} t\right) .
$$

It defines impulse response of the plate with frequency of harmonic vibration equal to resonant frequency of the plate.

Taking into account relation (10), phase changes of high frequency signal after passing $n$-th sensor is as follows:

$$
\varphi_{n}(t)=B_{n}(t) \mathrm{e}^{-\frac{\omega_{0}^{2} \tau}{2} t} \sin \left(\omega_{r n} t\right),
$$

where $B_{n}(t)$ - function defining force of $n$-th sensor. Functions $B_{n}(t)$ of each sensor are calculated from relation (13).

Phase shift of high frequency signal - relation (16) is time dependent function with resonant frequency of piezoelectric plate and its amplitude depends on force and damping. If external force decays, it is a function free damped vibration of the plate.

\section{Signal processing analysis of the output phase detector signal}

As was mentioned above in relations (6) and (7), phase shift of high frequency signal is a sum of phase shifts of all sensors in the system - Fig. 3. Phase shift of the single sensor is defined by relation (16). For linear range of phase detector - relation (5) - total output signal is

$$
U_{w y}(t)=\sum_{n=1}^{5} B_{n}(t) \mathrm{e}^{-\frac{\omega_{0}^{2} \tau}{2} t} \sin \left(\omega_{r n} t\right) .
$$

It is a sum of phase shifts from five sensors of the system - Fig. 3. Resonant frequencies localize sensors in 
a system and their amplitudes depend on type of force, which affects sensors. All sensors resonant frequencies are known. Thus only vibration amplitude of a sensor gives information about possible threat and electronic warning system responds if arbitrary selected amplitude threshold level is exceeded. Multichannel analog filters or digital filters with FFT could be applied to phase detector signal for standard output analysis. Considering multichannel analog filters application, any of filters have to be tuned to resonant frequency of particular SAW-VSs. Transfer function of each channel should secure sensor's distinguishability and maximize signal to noise ratio. These two conditions are fulfilled by matched filter, i.e.

$$
H(\omega)=S^{*}(\omega),
$$

where $H(\omega)$ - transfer function of channel, $S(\omega)$ - frequency response of the SAW-VS, * - complex conjugation.

It rises two problems to solve:

1. type of external force function is not known,

2. electronic parameters of the DAQ system should be find to acquire decaying signal from SAW-VS.

First problem implicates that output signal from phase detector and its frequency characteristic is unknown. However, relation (13) shows that this signal depends on external force and SAW-VS properties. The SAW-VS transfer function could be found from relation (11) or as a Fourier transform its impulse response defined by relation (15). Its amplitude transfer function is as follows:

$$
H(\omega)=\frac{a}{\sqrt{\left(1-\left(\omega / \omega_{0}\right)^{2}\right)^{2}+(\omega \tau)^{2}}} .
$$

Figure 4 shows typical amplitude of transfer functions for three different SAW-VSs.

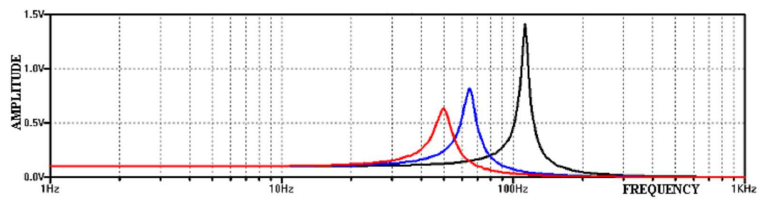

Fig. 4. Amplitude transfer functions for three different SAW-VSs.

As was expected amplitude transfer functions have special shape with higher values concentrated around resonant frequency. Relations (12) and (14) allow matching plates geometries. If resonant frequencies SAW-VSs are far from possible frequency characteristic of threat, then this characteristic does not influence output phase detector signal. Thus to solve first problem one has to find answers: what are resonant characteristics of SAW-VSs and what are differences between them. However, dynamic strength of plate $[3,4]$ and possibility to acquire signals form SAW-VSs have to be considered. This leads to second problem connected with DAQ system parameters. To define these parameters in Fig. 5 there are shown impulse responses of three SAW-VSs from Fig. 4.

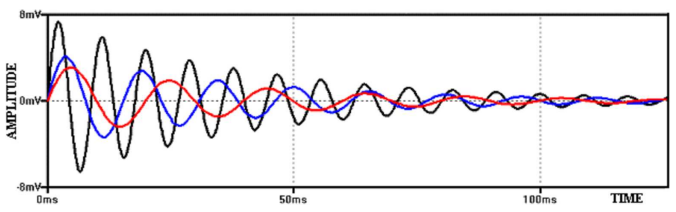

Fig. 5. Impulse response of three SAW-VS with amplitude transfer function from Fig. 4.

Sum of signals from the above figure is the output phase detector signal and it represents three SAW-VSs responses for the Dirac delta function of external force. In real environmental conditions impulse response of any SAW-VS is longer than showed in Fig. 5. Thus bandwidth of phase detector is narrower than bandwidth of amplitude transfer function of the SAW-VS. This means that real signal is easier to acquire than impulse response of sensor. Summarizing, multichannel filters and DAQ system are defined by impulse responses of SAW-VSs in the system.

\section{Conclusions}

Above presented analysis shows that electronic warning system based on SAW-VS is feasible. It was assumed that phase shifter works in linear range. Nowadays, linear characteristic of the phase detectors from relation (5) for signals up to $100 \mathrm{MHz}$ ranges from $-\pi / 6$ to $\pi / 6$ around working point. It induces necessity input signal phase shifting to the working point and this is a practical problem. All sensors in the system are wires connected, which involves constant delays in high frequency signal. Because total signal delay can shift working point of phase detector beyond its linear characteristic thus in each path of the system (Fig. 3) these additional phase shifters have to be removed from signals. It has to be done by phase shifters which allow adjusting phase difference of input signals of the phase detector to $\pi / 2$ value, while all SAW-VSs are fixed. Block diagram of such electronic warning system was shown in Fig. 6. It consists of five SAW-VSs placed in two lines, HFG, phase shifter (PS), PD, multichannel filter (MF), DAQ system.

Results of presented above analysis of electronic warning system are relatively simple relations defining design method of the SAW-VS resonant frequency. These frequencies should be higher than frequency response of external force which excites sensors. Mechanical vibrations range about $20 \mathrm{~Hz}$. Thus resonant frequencies should range from $20 \mathrm{~Hz}$ to $150 \mathrm{~Hz}$. Lower frequency is limited by size of the monocrystal wafer. Upper frequency is limited by time decay of vibrations and dynamic stress of piezoelectric plate. These limits depend on sensor architecture and electronic detection unit parameters. Simple description SAW-VS parameters allows us finding ex- 


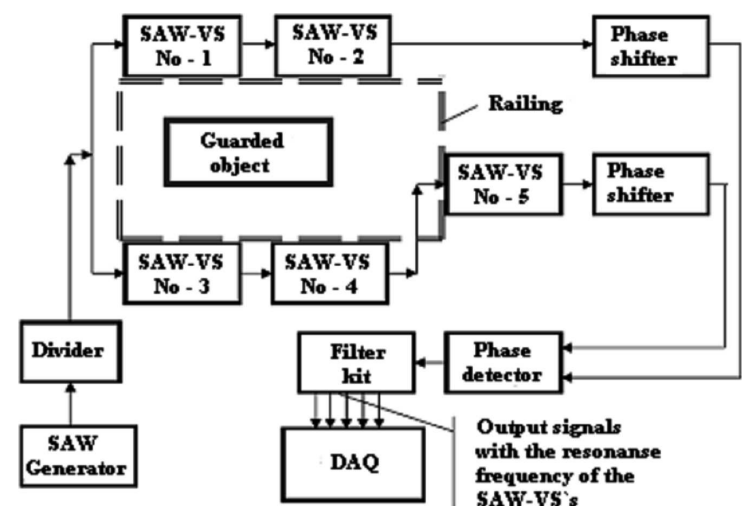

Fig. 6. Block diagram of electronic warning system with phase shifters and electronic detection unit.

plicit relations, which describe output phase detector signals and define multichannel filters characteristics and DAQ system parameters.

\section{Acknowledgments}

This work is supported by the Polish Ministry of Science and Higher Education as a research project "Vibrational electronic warning system with SAW sensors" implemented in 2009-2011.

\section{References}

[1] J. Filipiak, L. Solarz, G. Steczko, Acta Phys. Pol. A 116, 302 (2009).

[2] J. Filipiak, L. Solarz, K. Zubko, Mol. Quant. Acoust. 25, 89 (2004).

[3] J. Filipiak, Acceleration Sensors with Surface Acoustic Waves, Vol. 121, Częstochowa University Technology, Częstochowa 2006, p. 1.

[4] J. Filipiak, L. Solarz, G. Steczko, Mol. Quant. Acoust. 28, 71 (2007).

[5] T. Hejczyk, M. Urbanczyk, Acta Phys. Pol. A 118, 1153 (2010).

[6] J. Filipiak, L. Solarz, G. Steczko, Mol. Quant. Acoust. 28, 81 (2007) 